\title{
Abnormal expression of PTEN and PIK3CA in pemetrexed-resistant human pancreatic cancer cell line Patu8988
}

\author{
X. Shi, H.T. Gu, S.B. Lin, Y. Zhang, J. Yang and C.J. Qian \\ Department of General Surgery, \\ Affiliated Zhongda hospital of Southeast University, Gulou, Nanjing, Jiangsu, \\ China \\ Corresponding author: X. Shi \\ E-mail: shixined@126.com \\ Genet. Mol. Res. 15 (3): gmr.15036991 \\ Received August 12, 2015 \\ Accepted November 19, 2015 \\ Published July 29, 2016 \\ DOI http://dx.doi.org/10.4238/gmr.15036991
}

Copyright (C) 2016 The Authors. This is an open-access article distributed under the terms of the Creative Commons Attribution ShareAlike (CC BY-SA) 4.0 License.

\begin{abstract}
The aim of this study was to investigate the expression of PTEN and PIK3CA in the pemetrexed-resistant human pancreatic cancer cell line Patu8988, and to evaluate their effects on the biological behavior of pancreatic cancer cells. PTEN and PIK3CA gene and protein expressions were detected by reverse transcriptase polymerase chain reaction (RT-PCR) and western blot, respectively, in a pemetrexed-resistant pancreatic cancer cell line and in the parent strain of the pancreatic cancer cells. The discrepancies between the two types of cell lines were detected by a transwell test. RT-PCR and western blot analyses revealed that PTEN and PIK3CA were overexpressed in the pemetrexed-resistant pancreatic cancer cell line. PTEN and PIK3CA were shown to be upregulated by 89 and $76 \%$ (western blot), respectively, in the pemetrexed-resistant cell line, compared to the normal pancreatic cancer cell line. The migratory and invasive abilities
\end{abstract}


of the pemetrexed-resistant pancreatic cancer cell were significantly reduced compared to those of the parent strain $(\mathrm{P}<0.05$; transwell assay). Both PTEN and PIK3CA expression was abnormally enhanced in the pemetrexed-resistant cell line Patu8988; the co-existence of high levels of PTEN and PIK3CA in the pemetrexed-resistant pancreatic cancer line cells induced a significant decrease in their migratory and invasive capacities. This suggested that the mechanism of pemetrexed resistant may be affected by PTEN and PIK3CA, and that these may alter the biological behavior of cancer cells.

Key words: PTEN; PIK3CA; PI3K/Akt signal transduction pathway; Pancreatic cancer; Acquired drug-resistance; Pemetrexed

\section{INTRODUCTION}

Pancreatic cancer, a common alimentary malignant tumor, is the fourth leading cause of cancer mortality, with an increase in incidence rate over the years (Jemal et al., 2009). Acquired drug resistance is the major cause of chemotherapy failure in pancreatic cancer patients, similar to most other types of cancer. Pemetrexed is a multi-target anti-folate that functions as an anti-cancer agent by hampering the synthesis of purine and pyrimidine via the inhibition of various metabolic enzymes. Recent studies have identified the use of pemetrexed as a standard second-line chemotherapy regimen for patients with gemcitabine-refractory advanced pancreatic cancer, as it can significantly prolong patient survival and improve disease prognosis (Boeck et al., 2007).

Phosphatase and tensin homolog deleted on chromosome 10 (PTEN), also as well as mutated in multiple advanced cancer1 $(M M A C 1)$ and telomerase protein component 1 (TEP1), is one among the first tumor-suppressor genes to be identified and characterized. It is a dual-specificity phosphatase that dephosphorylates phosphatidylinositol-3,4,5trisphosphate to phosphatidylinositol-4,5-bisphosphate; therefore, it is a potent antagonist of the phosphoinositide-3-kinase (PI3K)/ATP-dependent tyrosine kinase (Akt) signaling transduction pathway. Mutations or deletions of PTEN activate the Akt pathway, leading to cancerization of the cell (Downes et al., 2007). Mutations and amplifications of PIK3CA, which enhances the phosphoinositide-3-kinase catalytic $\alpha$ subunit, also lead to the activation of the PI3K/Akt pathway.

However, so far, very few studies have focused on the expression of PTEN and PIK3CA in drug-resistant pancreatic cell lines, as well as their effect on the migratory and invasive abilities of pancreatic cells. The aim of this study is to determine the levels of expression of PTEN and PIK3CA in a pemetrexed-resistant human pancreatic cancer cell line and to gain some insight into the underlying drug resistance mechanism in pancreatic cancer.

\section{MATERIAL AND METHODS}

\section{Cell line and culture conditions}

The pemetrexed-resistant human pancreatic cancer cell line Patu8988 was provided by Dr. Yan (Yan et al., 2009), Medical College of Southeast University, who had previously

Genetics and Molecular Research 15 (3): gmr.15036991 
successfully induced and established the line. Materials required for cell culture, including high glucose Dulbecco's modified Eagle's medium (DMEM; Gibco; Life Technologies, Carlsbad, CA, USA), fetal bovine serum (FBS; Hangzhou Sijiqing Biological Material Co, Hangzhou, China), trypsinase (Gibco), were obtained. TRIzol reagent (for RNA extraction) and the required primers were obtained from Invitrogen (Grand Island, NY, USA). The reverse transcriptase polymerase chain reaction (RT-PCR) kit was obtained from Takara Bio Inc. (Tokyo, Japan). Phospho-PTEN (Ser-380/Thr-382/-383) rabbit mAb, rabbit anti-human PIK3CA mAb, and the secondary antibody (goat anti-rabbit IgG) were purchased from Cell Signaling Technologies (Beverley, MA, USA). Boster (Wuhan, China), and Santa Cruz Biotechnology (Santa Cruz, CA, USA), respectively. The western blot kit was purchased from Beyotime (Shanghai, China), while the Transwell plates and Matrigel were purchased from Becton, Dickinson Company (Franklin Lakes, NJ, USA).

\section{Cell culture}

The cells were divided into four groups: parent strain of the pancreatic cancer cell line Patu8988 (-), cells induced once with pemetrexed (+), cells induced twice with pemetrexed $(++)$, and cells induced thrice with pemetrexed $(+++)$. The half maximal inhibitory concentration of the cells induced with the drug for the third time was found to be 2.2 times greater than that of the parent strain $(\mathrm{P}<0.05)$, which indicated the successful induction of drug-resistance; the cell line was therefore defined as a pemetrexed-resistant cell line (Yan et al., 2009). All cells were incubated in high glucose DMEM supplemented with $10 \% \mathrm{FBS}$ at $37^{\circ} \mathrm{C}$ and a humidified atmosphere of $5 \% \mathrm{CO}_{2}$ in air. The culture solution was replaced on every alternate day; the cells were passaged after they grew to form a confluent layer; this layer was disrupted by trypsin $(2.5 \mathrm{~g} / \mathrm{L})$ digestion.

\section{Reverse transcriptase-polymerase chain reaction (RT-PCR)}

Total RNA was isolated using the TRIzol reagent, according to the manufacturer instructions. The reverse transcriptase reaction was performed in a total volume of $20 \mu \mathrm{L}$. The RT reaction conditions were set as follows: incubation at $42^{\circ} \mathrm{C}$ for $30 \mathrm{~min}$, heating at $99^{\circ} \mathrm{C}$ for $5 \mathrm{~min}$, cooling at $5^{\circ} \mathrm{C}$ for $5 \mathrm{~min}$, centrifuging approximately when the speed reaches the 6000-7000 rpm stop. The RT reaction product was subjected to immediate amplification, or stored at $-20^{\circ} \mathrm{C}$. The Primer premier (v.5.0, Premier, Canada) software was used to design the primer, and the primer specificity was verified by Basic Local Alignment Search Tool (BLAST) searching. The following conditions were applied for PCR amplification of the PTEN gene: pre-denaturation at $94^{\circ} \mathrm{C}$ for $2 \mathrm{~min}, 28$ cycles of denaturation at $94^{\circ} \mathrm{C}$ for $30 \mathrm{~s}$, renaturation at $61.8^{\circ} \mathrm{C}$ for $30 \mathrm{~s}$, extension at $72^{\circ} \mathrm{C}$ for $1 \mathrm{~min}$, and a final extension at $72^{\circ} \mathrm{C}$ for $7 \mathrm{~min}$, before termination of the reaction at $4^{\circ} \mathrm{C}$. Five microliters of the PCR products was subjected to routine electrophoresis in a $1.5 \%$ agarose gel $(100 \mathrm{~V}, 40 \mathrm{~min})$, in order to identify the amplified products. The primer sequences are summarized in Table 1.

\section{Western blot}

Total protein was extracted from the cells and quantified. Cell lysates of the four groups of cells were prepared and separated by polyacylamide gel electrophoresis (PAGE);

Genetics and Molecular Research 15 (3): gmr.15036991 
Table 1. Primer sequences for reverse transcriptase polymerase chain reaction (RT-PCR).

\begin{tabular}{|c|c|c|c|}
\hline Gene & Direction & Primer $\left(5^{\prime} \rightarrow 3^{\prime}\right)$ & Length of the product (bp) \\
\hline \multirow[t]{2}{*}{ PTEN } & + & 5'-CAT AAC GAT GGC TGT GGT TG-3' & \multirow[t]{2}{*}{347} \\
\hline & - & 5'-ACA TCT GAT TGG GAT GAG GC-3' & \\
\hline \multirow[t]{2}{*}{$P I K 3 C A$} & + & 5'-GTC AAT CGG TGA CTG TGT GG-3' & \multirow[t]{2}{*}{354} \\
\hline & - & 5'-GTC AAA ACA AAT GGC ACA CG-3' & \\
\hline \multirow[t]{2}{*}{$\beta$-actin } & + & 5'-ATC CAT CCG ACA TTG AAG T-3' & \multirow[t]{2}{*}{155} \\
\hline & - & 5' -CCT TCA CCG TTC CAG TTT-3' & \\
\hline
\end{tabular}

the separated proteins were transferred onto a nitrocellulose membrane (Biyuntian, Nantong, China), which was incubated overnight at $4^{\circ} \mathrm{C}$ with the following primary antibodies: PTEN first antibody (1:1000 in TBST) and PIK3CA antibody (1:200 in TBST). The membrane was then incubated with the respective secondary AP-labeled IgG antibodies at $26^{\circ} \mathrm{C}$ for $60 \mathrm{~min}$. The membrane was stained and observed by ECL; the gray scale value was analyzed using the Quantity One software (BIO-RAD, USA).

\section{Migration and invasion assays}

Cell invasiveness was analyzed using a Matrigel-coated invasion chamber. The migratory and invasive capacities of the cells were determined by assaying cells treated at $37^{\circ} \mathrm{C}$ with a humidified atmosphere of $5 \% \mathrm{CO}_{2}$ in air for 6 and $24 \mathrm{~h}$, respectively. These were then fixed, and dried, and observed under a microscope (and subsequently photographed).

The data were statistically analyzed using Graph-Pad Prism 5.0. Differences between the two groups were analyzed a two-tailed Student $t$-test; P values $<0.05$ were considered significant. All data are reported as means \pm standard deviation.

\section{RESULTS}

\section{$P T E N$ and $P I K 3 C A$ expression evaluated by RT-PCR}

The expression of PTEN and PIK3CA differed significantly among the four groups of cells: the expression of PTEN and PIK3CA in the parent strain (-), cells induced once with pemetrexed $(+)$, cells induced twice with pemetrexed $(++)$, and those induced thrice with pemetrexed $(+++)$. That is, the expression of PTEN and PIK3CA was the highest in pemetrexedresistant pancreatic cancer cells $(+++)$ and the lowest in the parent strain (-), as seen in Figure 1.

\section{PTEN and PIK3CA protein expression evaluated by western blot}

The expression of PTEN and PIK3CA was higher in the cells of the drug-induced groups than in the normal pancreatic cancer cells, which reflected the results of the RT-PCR analysis. PTEN and PIK3CA expression was upregulated by 89 and $76 \%$, respectively, in cells induced thrice with pemetrexed $(+++)$ (Figure 2).

\section{Migration and invasion assays}

The results of the in vitro migration and invasion assays indicated an obvious decrease in the migratory and invasive capacities of the cells in the drug-induced groups $(28.93 \pm 3.01$ 
and $37.73 \pm 2.55$, respectively), compared to that observed in the normal pancreatic cancer cells, (48.73 \pm 4.89 and $54.87 \pm 4.16$, respectively; $\mathrm{P}<0.05$; Figure 3$)$.
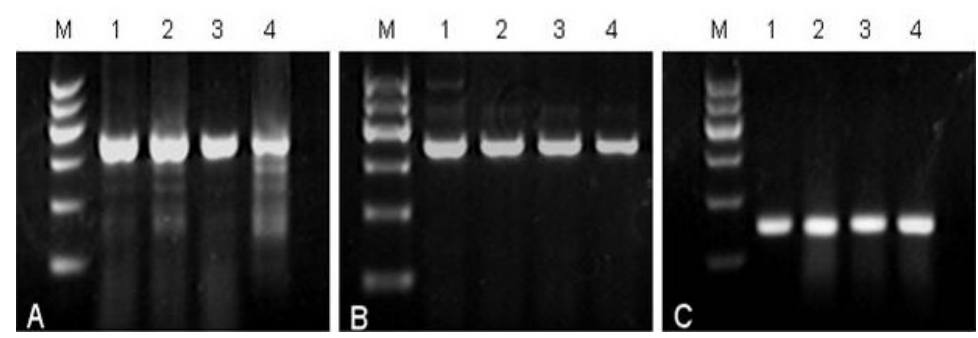

Figure 1. Reverse transcriptase polymerase chain reaction (RT-PCR) to detect the expression of PTEN and PIK3CA in cells induced with pemetrexed and the parent strain. A. PTEN (347bp); B. PIK3CA (354bp); C. $\beta$-actin (155 $\mathrm{bp})$; lane $M=$ marker $(100,200,300,400,500$, and $600 \mathrm{bp})$. Lane $1=$ pemetrexed-resistant pancreatic cancer cells $(+++)$; lane 2 = cells induced twice with pemetrexed $(++)$; lane 3 = cells induced once with pemetrexed $(+)$; lane $4=$ parent strain of Patu8988.

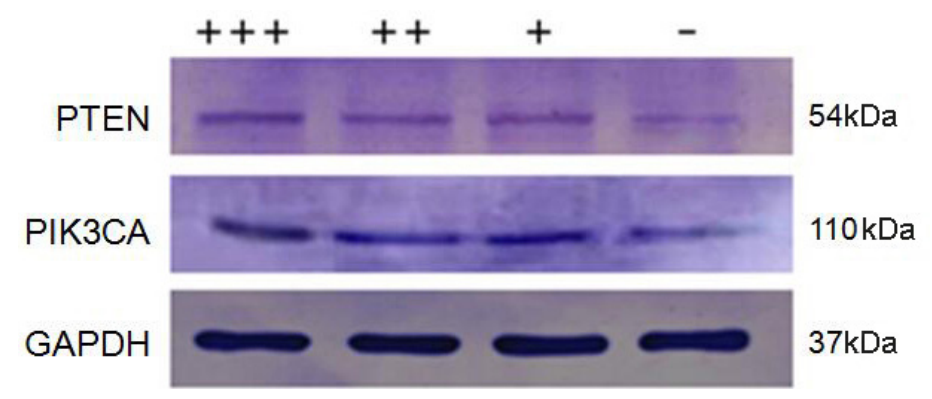

Figure 2. Western blot to detect the expression of PTEN and PIK3CA in cells induced with pemetrexed and in the parent strain. The top, middle, and bottom rows indicate the expression of PTEN, PIK3CA, and GAPDH proteins in pemetrexed-resistant pancreatic cancer cells $(+++)$; cells induced twice with pemetrexed $(++)$; cells induced once with pemetrexed (+); and the parent strain of Patu8988.
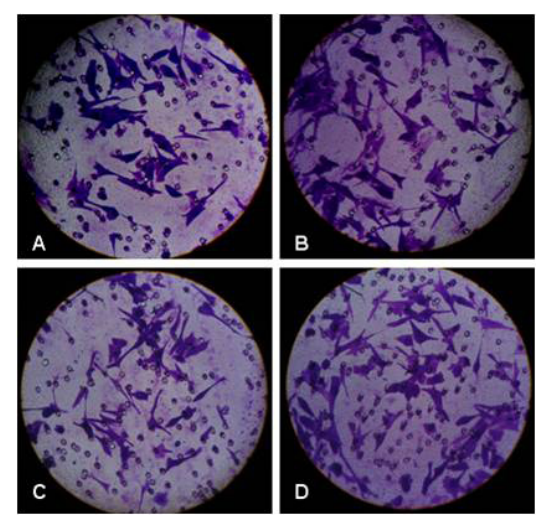

Figure 3. Results of the migration and invasion assays in pemetrexed-resistant pancreatic cancer cells and in cells of the parent strain (400X). A. B. Migration assays. C. D. Invasion assays. A, C: pemetrexed-resistant pancreatic cancer cell Patu8988; B, D: the parent strain ofPatu8988.

Genetics and Molecular Research 15 (3): gmr.15036991 


\section{DISCUSSION}

The development of drug resistance by cancer cells is the major cause of chemotherapy failure. However, this mechanism remains relatively unknown. Recent studies have discovered that in addition to affecting tumorigenesis and cell growth, the PI3K/Akt signal transduction pathway plays a role in the development of chemoresistance (Fresno Vara et al., 2004). Previous studies have also shown that the use of chemotherapeutic agents in combination with small molecule inhibitors of the kinases involved in this pathway, such as Wortmannin and LY294002, can augment the sensitivity of cancer cells to anti-cancer drugs (Kim et al., 2007; Li et al., 2008; LoPiccolo et al., 2008); this suggests that the PI3K/Akt pathway may be involved in the mechanism underlying chemoresistance. Mutations in or a deletion of PTEN, a negative regulator of the PI3K/Akt pathway, can enhance the PI3K/Akt pathway, causing drug resistance. In this study, the expression of PTEN was higher in the pemetrexedresistant pancreatic cancer cell line Patu8988 than in the parent strain, which suggested the existence of mechanism that blankets the PTEN activity or enhances the activity of PI3K-via an as-yet-unclear pathway, thereby maintaining a normal expression of PTEN. This could via the following potential mechanisms: 1) Decrease in PTEN recruitment on the cell membrane: PTEN functions by binding with PI $(3,4,5)$ P3, after de-phosphorylation on the cell membrane. Therefore, impairment of PTEN activity would decrease its capacity to bind with PI $(3,4,5)$ P3, resulting in the inhibition of its activity; 2) The existence of a potential factor that suppress PTEN activity. DJ-1 was first reported as a potential suppressor of PTEN by Kim et al. (2005) subsequent studies have shown that PTEN could also be negatively regulated by other factors. 3) Mutations in the PTEN gene: PTEN is generally expressed in normal amounts or over-expressed; changes in the amino acid structure resulting from mutations in the PTEN gene could lead to its complete deactivation; 4) Compensated expression: hyperexpression of the downstream factors of PTEN, such as PIK3CA and Akt, would result in the overexpression of PTEN.

A high frequency of mutations in the PIK3CA gene, which encodes the catalytic subunit pll0 $\alpha$ of I A PI3K, has been observed in many types of human malignant tumors (Samuels et al., 2004). In addition, this gene is amplified and overexpressed in different types of cancers. Therefore, PIK3CA has been the subject of intensive research over the past few years. In this study, the drug-resistant pancreatic cancer cell line showed higher PIK3CA expression than that in the parent strain, which corresponded with the development of C-DDP resistance in hypo-PIK3CA-expressed ovarian cancer cells (Lee et al., 2005). Therefore, PIK3CA was believed to be involved in the development of drug-resistance in pancreatic cancer cells.

It has been generally accepted that PTEN and PIK3CA cannot be expressed abnormally in the same tumor, which suggests that the presence of two pathways for regulation of carcinogenic genetics: the activation of PTEN and the activation of PIK3CA. Moreover, this also suggests the lack of any crosstalk between these two gene regulation pathways. However, PTEN and PIK3CA are both shown to be hypo-expressed in a drug-resistant pancreatic cancer cell line in this study, which indicated a collaborative effect of PTEN and PIK3CA overexpression on drug-resistance induced by the PI3K/Akt pathway in pancreatic cancer.

The mechanism of invasion and extension in malignant tumor is affected by cell heredity, surface structure, invasive power, and adhesive ability, among others. In this study, the invasive and migratory powers of a pemetrexed-resistant pancreatic cancer cell line that showed simultaneous hypoexpression of both PTEN and PIK3CA were significantly weakened compared to those of the parent strain $(\mathrm{P}<0.05)$. This could be attributed any of

Genetics and Molecular Research 15 (3): gmr.15036991 
the following mechanisms: 1) PTEN is a tumor-suppressor gene that can de-phosphorylate lipoids: Under normal circumstances, PTEN hampers PI3K and functions as a negative regulator of Akt activity, which helps maintain normal Akt activity. PTEN protein may also impair the transcription power of nuclear factor $\kappa \mathrm{B}(\mathrm{NF}-\kappa \mathrm{B})$ by down-regulating the activity of PI3K/Akt, and positively regulating the invasive and migratory powers of the cells by decreasing-matrix metalloproteinase expression; 2) the integrin-induced aggregation and adhesion process is affected by PTEN-induced de-phosphorylation of focal adhesion kinase, which in turn inhibits the infiltration and metastasis of tumor cells (Osaki et al., 2004); 3) A comparison of the drug- resistant pancreatic cells with the parent strain revealed that induction of drug resistance may impair the motor capacity of pancreatic cells.

In this study, we observed the abnormal expression of PTEN and PIK3CA in a pemetrexed-resistant pancreatic cancer cell line. They may have a concomitant effect on the resistance of the pancreatic cancer cells to pemetrexed, which suggested that the PI3K/ Akt signal transduction pathway may play a role in the development of drug resistance in pancreatic cancer cells. The effect of these two genes on the response to chemotherapy must be ascertained by further studies, using methods such as siRNA silencing, and plasmid transfection. We also demonstrated that the co-existence of high levels of PTEN and PIK3CA induced a significant decrease in the migratory and invasive capacities of pemetrexed-resistant pancreatic cancer cells. The role of the PI3K/Akt pathway in acquired drug-resistance must be elucidated and specific inhibitors of this pathway must be explored to develop methods to successfully address pancreatic cancer drug-resistance in the future.

\section{Conflicts of interest}

The authors declare no conflict of interest.

\section{ACKNOWLEDGMENTS}

Research supported by the National Natural Science Foundation of China (\#30772541).

\section{REFERENCES}

Boeck S, Weigang-Köhler K, Fuchs M, Kettner E, et al. (2007). Second-line chemotherapy with pemetrexed after gemcitabine failure in patients with advanced pancreatic cancer: a multicenter phase II trial. Ann. Oncol. 18: 745751.http://dx.doi.org/10.1093/annonc/mdl463

Downes CP, Perera N, Ross S and Leslie NR (2007). Substrate specificity and acute regulation of the tumour suppressor phosphatase, PTEN. Biochem. Soc. Symp. 74: 69-80. http://dx.doi.org/10.1042/BSS2007c07

Fresno Vara JA, Casado E, de Castro J, Cejas P, et al. (2004). PI3K/Akt signalling pathway and cancer. Cancer Treat. Rev. 30: 193-204.http://dx.doi.org/10.1016/j.ctrv.2003.07.007

Jemal A, Siegel R, Ward E, Hao Y, et al. (2009). Cancer statistics, 2009. CA Cancer J. Clin. 59: 225-249. http://dx.doi. org $10.3322 /$ caac. 20006

Kim RH, Peters M, Jang Y, Shi W, et al. (2005). DJ-1, a novel regulator of the tumor suppressor PTEN. Cancer Cell 7: 263-273.http://dx.doi.org/10.1016/j.ccr.2005.02.010

Kim SH, Juhnn YS and Song YS (2007). Akt involvement in paclitaxel chemoresistance of human ovarian cancer cells. Ann. N. Y. Acad. Sci. 1095: 82-89. http://dx.doi.org/10.1196/annals.1397.012

Lee S, Choi EJ, Jin C and Kim DH (2005). Activation of PI3K/Akt pathway by PTEN reduction and PIK3CA mRNA amplification contributes to cisplatin resistance in an ovarian cancer cell line. Gynecol. Oncol. 97: 26-34. http:// dx.doi.org/10.1016/j.ygyno.2004.11.051

Li H, Yu JP, Yu HG, Cao J, et al. (2008). Effect of PI3K/Akt inhibitor on the expression of Caspase-9 in human gastric cancer cells. Wuhan Daxue Xuebao Yixue Ban 29: 38-40.

Genetics and Molecular Research 15 (3): gmr.15036991 
LoPiccolo J, Blumenthal GM, Bernstein WB and Dennis PA (2008). Targeting the PI3K/Akt/mTOR pathway: effective combinations and clinical considerations. Drug Resist. Updat. 11:32-50.http://dx.doi.org/10.1016/j.drup.2007.11.003

Osaki M, Oshimura M and Ito H (2004). PI3K-Akt pathway: its functions and alterations in human cancer. Apoptosis 9: 667-676.http://dx.doi.org/10.1023/B:APPT.0000045801.15585.dd

Samuels Y, Wang Z, Bardelli A, Silliman N, et al. (2004). High frequency of mutations of the PIK3CA gene in human cancers. Science 304: 554. http://dx.doi.org/10.1126/science.1096502

Yan W, Shi X, Ge Z, Xiao Z, et al. (2009). The molecular biological mechanism of acquired resistance of pancreatic cancer cell to pemetrexed. J. Pract. Oncol. 23: 106-110.

Genetics and Molecular Research 15 (3): gmr.15036991 